current study identified risk factors for the acquisition of these MDR gram-negative critical priority pathogens in King Abdulaziz Medical City, Jeddah, to inform strategies for their containment.

Methods A case-control study was carried out from January to April 2015, in which 100 patients with healthcare-associated infections (infections arising 48 hours after admission) caused by MDR GNB were compared with two control groups, i.e., 100 patients with healthcare-associated infections caused by non-MDR GNB (not meeting the criteria of MDR) and 100 patients without infection caused by GNB. MDR bacteria were defined as bacteria that were non-susceptible to at least one antibiotic in three or more classes of antibiotics. Data were analyzed using descriptive statistics (frequency and percentage of categorical variables). Multivariate regression analysis was undertaken to identify significant predictors of MDR GNB. Odds ratios (ORs) with 95\% CIs were calculated and the level of significance was determined as $\mathrm{p}<0.05$.

Results A total of 388 organisms were isolated during the study period from 332 patients. 56 (14\%) patients were infected with more than one organism. Antibiotic therapy (OR 5.50, 95\% CI 2.19-13.84; OR 3.98, 95\% CI 1.68-9.44), stay in intensive care unit (OR 11.11, 95\% CI 4.58-26.93; OR 8.60, 95\% CI 3.28-22.57), and having indwelling medical devices (OR 3.02, 95\% CI 1.45-6.33; OR 2.43, 95\% CI 1.11-5.33) were the significant risk factors in patients infected with MDR GNB compared with each of the other two control groups, respectively.

Conclusion The risk factors identified in our study provide guidance for healthcare workers for the prevention and containment of MDR GNB with special emphasis on effective implementation of an antimicrobial stewardship program and enhancing infection control practices in intensive care units.

\section{THE EFFECTIVENESS OF STERILE TECHNIQUE IN REDUCING FALSE-POSITIVE BLOOD CULTURE RESULTS IN QATIF CENTRAL HOSPITAL EMERGENCY DEPARTMENT}

Mohammed AL-Bazroun, Auday ALKhunaizi, Arif Al-Hamad, Ali Ismail, Zahra Talib, jaffar AL- Twoailb, Maha al Ibrahim, M Alquraini. Emergency Department/Laboratory Department Qatif Central Hospital

\subsection{6/bmjoq-2019-PSF.36}

Background Blood culture contamination is a common and preventable problem in the emergency department (ED). In previous studies, changing the process of ED blood culture collection into a more sterilized procedure $r$ esulted in a substantial reduction in the rate of blood contamination. The present study assessed the degree of blood contamination and evaluated the effect of using a sterile technique with monitoring and feedback on contamination rate over a 1-year period.

Methods We documented the rate of blood contamination among blood samples sent from the ED in the period from January 2016 until March 2016. A workshop for all ED nurses was held in March 2016 by clinical nurse instructors and this was followed by daily bedside teaching sessions for the whole study period. Nurses were instructed and audited on proper sterile blood withdrawal techniques. During the intervention period, we measured the rate of blood contamination for the period from April 2016 until September 2016. Results Our average contamination rate dropped from the baseline of $12.6 \%$ (58 out of a total of 736 samples) to an average contamination rate of approximately 5.6\% (122 out of a total of 1549 samples), with an odds ratio of 0.411 (95\% CI 0.303-0.559; p<0.001).

Conclusion Changing the method of blood culture collection from the commonly used aseptic technique to a sterile process resulted in significant reductions in blood culture contamination in a busy community hospital ER. Monitoring the implementation process was important to identify and overcome operational challenges. In addition, this study could be a good initiative to start a multicentric quality improvement project to reduce blood contamination in the neighboring community and public hospitals.

\section{MEDICATION EXCHANGE AND SHARING NETWORK PROGRAM (MESNP) INITIATIVE TO COPE WITH DRUG SHORTAGES IN THE KINGDOM OF SAUDI ARABIA (KSA)}

Aeshah AlAzmi, Rph Faris AlRashidi. Pharmaceutical Care Services Department, King Abdulaziz Medical City Jeddah

\subsection{6/bmjoq-2019-PSF.37}

Background Drug shortages are a major public health concern and remain a persistent problem worldwide. Saudi Arabia is one of the richest and fastest growing countries in the Middle East. Despite that, Saudi Arabian drug markets are not immune to drug shortages. Although exact figures about drug shortages in Middle Eastern countries in general and in Saudi Arabia, in particular, are lacking, there is an emerging yet still limited number of reports about the drug shortage. It is, however, a fact that the drug shortages are affecting the Middle East in general and the Kingdom of Saudi Arabia (KSA) in particular. At the time we conducted this project, the Saudi Food and Drug Administration (SFDA) had not yet fully activated and implemented its role in tracking drug shortages and the role of other regulatory bodies were either outdated or unknown. Healthcare is one of the main focus areas of Saudi Vision 2030, which represents a comprehensive plan for the entire economic structure of Saudi Arabia. In order to ensure the Saudi Vision 2030 becomes reality, we should focus on more efficient use of our current resources. Based on that, we identified an innovative solution at the national level to collaborate and cope with the current situation by developing a centralized Medication Exchange and Sharing Network Program (MESNP).

Methods A quality improvement process map method was used for this project. Baseline evaluation included a review of possible reasons and strategies to manage medication shortages, recognize potential associated safety issues, and we developed MESNP as a national novel project to cope with medication shortages using a telegram as the preferred social media platform for group creation and communication.

Results A total of 500 reports were received. The majority of reports $(70 \%)$ were raised by the Ministry of Health $(\mathrm{MOH})$. A number of reports constituted requests for drug supplies due to shortages $(n=315)$ and reports indicating the availability of overstock items for redistribution $(n=185)$. Almost 98\% of overstocking drug reports were redistributed, which covered $75 \%$ of drug shortage requests.

Conclusion We believe that this is the first national novel project aiming to address drug shortages. The optimistic findings of this project were the proactive identification of data and development of a framework to collect data about 
national drug shortages to facilitate the medication exchange and sharing between organizations to prevent drug wastages and shortages for better patient care.

\section{IDENTIFICATION OF THE INCIDENCE OF ADVERSE DRUG REACTIONS (ADRS) USING NALOXONE AS A TRIGGER TOOL: A RETROSPECTIVE ANALYSIS}

Nissreen Althaqafi, Asmaa Battah, Eman Youssif. Pharmaceutical Care Services Department, King Abdulaziz Medical City Jeddah

\subsection{6/bmjoq-2019-PSF.38}

Background Adverse drug reactions (ADRs) adversely affect patient outcomes, which may cause patients to lose confidence in the healthcare system. Even with the drastic improvement in healthcare practices, detection of ADRs continues to be an important safety tool to ensure patient treatment outcome and safety. The Institute for Healthcare Improvement (IHI) developed a trigger tool as a method to identify possible adverse events from medicine use in the inpatient setting. In this context, we identify naloxone as a trigger tool to detect unreported adverse effects secondary to the use of medicine.

Methods A retrospective chart review of naloxone prescribed to all admitted patients at KAMC-J over 1 year (2016 to 2017) was done to assess the trigger tool efficacy in the identification of ADRs and to assess the appropriate use of naloxone. We defined the appropriate use of naloxone as documentation of the reason for ordering being present and appropriate. The other objective was to determine the proportion of incidents documented through the safety reporting system (SRS).

Results A total of 100 patients who received naloxone orders were identified, for which all were administered in the inpatient setting. The majority of naloxone orders $(n=62,62 \%)$ were to reverse mental status changes, while six patients $(6 \%)$ required intensive care admission. Only four (4\%) cases out of 100 had a documented ADR report through the SRS. The most commonly prescribed dose of naloxone was $0.4 \mathrm{mg}$ $(56 \%)$ followed by $0.2 \mathrm{mg}(23 \%)$. Only two patients received a higher initial dose of $2 \mathrm{mg}$. The majority were prescribed secondary to morphine (IV) or fentanyl (IV or patch), or hydromorphone (PO in patient with end-stage renal disease), and three patients received naloxone secondary to benzodiazepine administration. Two geriatric patients received naloxone without clear justification and they were not on any opioid drugs. The rest of the patients received various doses $(0.04$ to $1.2 \mathrm{mg})$.

Conclusion Using naloxone as a trigger tool is effective in tracing and tackling ADRs in our institution. We found that naloxone administration was often inappropriate. The most common order for naloxone was a $0.4 \mathrm{mg}$ IV push dose, which caused a reversal of analgesia. Development of guidelines and order sets defining the appropriate use of naloxone will help guide healthcare providers on the appropriate ordering of naloxone based on the clinical situation. Although serious ADR reports are minimal in our data (6\%), it did not eliminate the chance of missing important serious reports due to under-reporting.

\section{9 IMPLEMENTING LARGE-SCALE CHANGE OF QUALITY CULTURE: ABU DHABI STORY}

Asma Alameri, Asma Almannaei, Bashir Aden, Jumana Alabed. Healthcare Quality, Department of Health - Abu Dhabi

\subsection{6/bmjoq-2019-PSF.39}

Background The Department of Health - Abu Dhabi (DoH) is the responsible organization that ensures high-quality, sustainable, and accessible care to a population of about 3 million. Improving the quality of care and enhancing patient experience are the top strategic priorities for the DoH and for Abu Dhabi Vision 2020. However, a quality framework had not been put in place to monitor the performance of healthcare providers and measure the quality levels. Moreover, there were variations in the clinical outcomes among the peer group of providers, which affected the patient experience. In order to improve the quality of care and patient experience, DoH introduced Abu Dhabi Quality Index in 2014, a comprehensive quality monitoring framework that is based on the latest thinking and innovative solutions that allows for continuous quality improvements. It consists of Jawda (quality in Arabic) indicators, patient experience survey, and healthcare professional satisfaction survey.

Methods To implement a wide scale of quality change, the DoH developed and implemented a quality improvement framework to enhance quality culture at system level, improve patient safety at the population level, and reduce quality outcome variation among all healthcare providers. This was achieved through a set of quality indicators, Jawda indicators, that measure clinical outcomes, patient safety, and accessibility. The initiation of what to focus on and the vison was around patients' complaints analysis, workshops with stakeholders, and one-to-one meetings. This was a key driving case for change.

Results The latest data collected from 45 hospitals in Abu Dhabi have shown the following results:

- $20 \%$ reduction in unplanned readmission rate for pneumonia.

- $50 \%$ reduction in cardiopulmonary arrest outside critical care.

- $40 \%$ reduction in rate of falls resulting in any injury.

- $30 \%$ reduction in hospital-acquired or worsening pressure ulcer rate.

- $50 \%$ reduction in emergency primary cesarean section rate.

- $40 \%$ reduction in surgical site infection rate for emergency cesarean section.

Conclusion It has been 4 years since the introduction of Jawda indicators to the health sector in Abu Dhabi, and there have been improvements on the average of quality performance and reduction in quality variation among hospitals. More importantly, quality culture, transparency, and accountability were enhanced. The latest improvements to the program include risk adjustments and clinical subspecialty indicators such as stroke, orthopedics, and bariatric surgeries. In 2019, new quality dimensions will be added to the Abu Dhabi Healthcare Quality Index and hospital rankings will be published. 